\title{
Diffusion Monte Carlo in Continuous Time
}

\author{
Olav F. Syljuåsen \\ NORDITA, Blegdamsvej 17, DK-2100 Copenhagen Ø, Denmark
}

A detailed outline of a diffusion Monte Carlo algorithm for lattice models is given. The method is particular in that it has no time discretization errors. It is applied to two models: 1) A lattice boson Hubbard model where the bosons carry an internal spin-1 degree of freedom, and 2) The quantum dimer model on the square lattice. In the latter deconfinement of monomers is demonstrated for monomer hopping strengths exceeding a critical value.

PACS numbers: 74.20.Mn, 75.10.Jm, 02.70.Ss

\section{INTRODUCTION}

Diffusion Monte Carlo(DMC) is perhaps the most intuitive and natural of the projection Monte Carlo techniques in that it aims at following the imaginary-time development of the ground state wave function ${ }^{1}$. Usually this evolution is performed stepwise in discrete time steps which causes a time discretization error. However for lattice models this evolution may be performed in continuous-time getting rid of this error ${ }^{2}$. The continuous-time formulation of DMC is applicable to any lattice models not suffering from the sign problem. We will demonstrate its applicability by giving results for two quite different models: A Hubbard model for antiferromagnetic spin-1 bosons and the quantum dimer model on the square lattice. Before embarking on the applications a detailed account of the algorithm will be given.

\section{ALGORITHM}

The basic idea behind DMC is to simulate the power method stochastically. The power method uses repeated matrix multiplications to project out the matrix eigenstate having the largest eigenvalue. In the special case of DMC the matrix used is the imaginary-time evolution operator which 


\section{O.F. Syljuåsen}

projects out the ground state of the Hamiltonian. Specifically

$$
e^{-\left(H-E_{0}\right) \tau}\left|x_{I}\right\rangle \stackrel{\tau \rightarrow \infty}{=} C_{x_{I}}\left|\psi_{0}\right\rangle
$$

where $H$ is the Hamiltonian, $E_{0}$ is the ground state energy and $\left|x_{I}\right\rangle$ is an arbitrary initial state having overlap $C_{x_{I}}=\left\langle\psi_{0} \mid x_{I}\right\rangle$ with the ground state $\left|\psi_{0}\right\rangle$.

In order to explain how the multiplication by $e^{-\left(H-E_{0}\right) \tau}$ is carried out in continuous-time, that is without discretizing $\tau$, it is helpful to consider the example of a two-state system with Hamiltonian matrix elements: $H_{i j}=\langle i|H| j\rangle$, where $i, j=\{1,2\}$. For this two-state system an instance of the (unnormalized) wave function is described by a two-dimensional vector having non-negative integer entries

$$
|\psi\rangle=\left(\begin{array}{c}
M_{1} \\
M_{2}
\end{array}\right)
$$

One can think of this integer-valued vector as representing a count of $M_{1}+M_{2}$ replicas or copies of the system, where $M_{1}$ of them are in state 1 and $M_{2}$ are in state 2. For big systems the dimension of the vector is exponentially large, and with a finite number of replicas it will be very sparse. Thus it is best to keep track of the state of each replica rather than writing down the vector explicitly. The requirement that the number of replicas in a given state is non-negative is rather restrictive, and is equivalent to the requirement that there is no sign problem, which will be assumed in the following.

In order to build up the continuous-time formulation we will consider the evolution for an infinitesimal time step and then explain how to piece together (infinitely) many of these time steps in one shot. The action of the evolution operator for an infinitesimal time step $d \tau$ on an instance of the state is

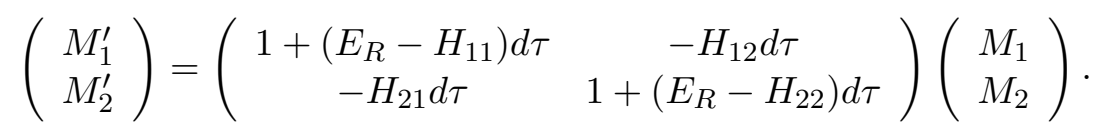

Note that the ground state energy $E_{0}$ is not known at the outset of the simulation. Therefore an estimator of the ground state energy commonly known as the reference energy $E_{R}$ is introduced and used instead. During the course of the simulation this reference energy will be adjusted and can be used to extract the ground state energy. One should note that a time varying $E_{R}$ causes the evolved wave function to deviate from the ground state wave function. This can be repaired by reweighting the simulation which has become a standard procedure used in $\mathrm{DMC}^{3}$.

We will now formulate a stochastic process that on average yields the evolution equation above. In the time interval $d \tau$ a replica in state $|i\rangle$ can undergo one out of four different processes with different probabilities: 


\section{Continuous-time Diffusion Monte Carlo Algorithm for Lattice Models}

- "Transition", change state to $|j\rangle, j \neq i$, probability: $P_{T j}(i)$.

- "Die", that is $M_{i} \rightarrow M_{i}-1$, probability: $P_{D}(i)$.

- "Replicate", that is $M_{i} \rightarrow M_{i}+1$, probability: $P_{R}(i)$.

- "Stay", stay unchanged in state $|i\rangle$, probability: $P_{S}(i)$.

As these are all possibilities, probability conservation implies

$$
P_{T j}(i)+P_{D}(i)+P_{R}(i)+P_{S}(i)=1,
$$

and must hold for all states $i=1,2$.

The task of identifying the probabilities with matrix elements of the Hamiltonian is easy. Because the off-diagonal matrix element $H_{j i}$ is the only one responsible for transition between state $i$ and $j$ it is clear that

$$
P_{T j}(i)=-H_{j i} d \tau
$$

where $j \neq i$. As usual on order to avoid the sign problem off-diagonal matrix elements are restricted to be negative, which will be assumed in the following. The increase in number of replicas in state $|i\rangle$ from processes acting on replicas in state $|i\rangle$ is

$$
M_{i}^{\prime}-M_{i}=\left[P_{R}(i)-P_{D}(i)-P_{T j}(i)\right] M_{i} .
$$

This implies when comparing to the diagonal elements of Eq. (3) and using Eq. (5) that

$$
P_{D}(i)-P_{R}(i)=\left(H_{i i}-E_{R}+H_{j i}\right) d \tau
$$

where $j \neq i$. The right hand side of the above takes either a positive or a negative value. In order to reduce the fluctuations in the replica numbers as much as possible $P_{R}=0$ is chosen whenever this value is positive and $P_{D}=0$ when it is negative. This choice implies that $P_{D}$ and $P_{R}$ are of the order $d \tau$ as also holds for $P_{T}$. The probability conservation equation Eq. (4) then implies

$$
P_{S}(i)=1-\left(\left|H_{i i}-E_{R}+H_{j i}\right|-H_{j i}\right) d \tau,
$$

which is of the order unity.

We are now at the stage where we wish to patch together many infinitesimal time steps. From the fact that $P_{S}$ is of the order unity and all other processes are of the order $d \tau$ it follows that for most (infinitesimal) time intervals nothing happens to each replica! This implies that the process can be modeled like the radioactive decay problem, although here with three different decay channels, "Transition", "Die", and "Replicate". This observation has been used previously to construct continuous-time algorithms for 


\section{O.F. Syljuåsen}

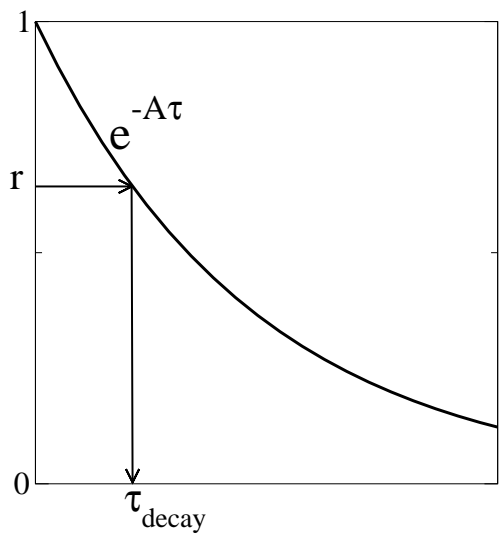

Fig. 1. Selecting decay times according to the exponential distribution with decay constant $A$. Drawing a random number $r$, the decay time $\tau_{\text {decay }}$ is selected as $\tau_{\text {decay }}=\frac{1}{-A} \ln (r)$.

finite-temperature quantum Monte Carlo methods that samples the quantum partition function ${ }^{4}$. Thus we can simulate the imaginary-time evolution of one replica by generating exponentially distributed decay times with decay constant $A=\left|H_{i i}-E_{R}+H_{j i}\right|-H_{j i}$, see Fig. 1 .

Having obtained the decay time, the type of decay is determined stochastically proportional to the respective probabilities $P_{T}, P_{D}$ and $P_{R}$, which are all of the order $d \tau$. As an example, if $P_{T}=T d \tau, P_{D}=D d \tau$ and $P_{R}=0$, the "Transition" process would be chosen with probability $T /(T+D)$ and "Die" with probability $D /(T+D)$.

The generalization to large systems is immediate. In Eq. (6) $P_{T j}(i) \rightarrow$ $\sum_{j \neq i} P_{T j}(i)$ to account for transitions to all possible states different from $|i\rangle$. It follows that in the many states case

$$
P_{D}(i)-P_{R}(i)=\left(H_{i i}-E_{R}+\sum_{j \neq i} H_{j i}\right) d \tau
$$

and

$$
P_{S}(i)=1-\left(\left|H_{i i}-E_{R}+\sum_{j \neq i} H_{j i}\right|-\sum_{j \neq i} H_{j i}\right) d \tau .
$$

The decay constant is thus $A=\left|H_{i i}-E_{R}+\sum_{j \neq i} H_{j i}\right|-\sum_{j \neq i} H_{j i}$. Instead of having just one "Transition" decay channel as was the case for the two-state system, the non-zero off-diagonal matrix elements on the $i$ 'th column of the Hamiltonian determines different "Transition" processes. Selecting among these is still easy as they are all of the same order $d \tau$ (as are $P_{D}$ and $P_{R}$ ). 


\section{Continuous-time Diffusion Monte Carlo Algorithm for Lattice Models \\ 3. SPIN-1 ANTIFERROMAGNETIC BOSE-HUBBARD MODEL}

The development of cooling techniques for atoms in magnetic and optical traps have made it possible to study bosonic Mott insulators of atoms ${ }^{5}$. While many details are known about the Bose-Hubbard model describing trapped atoms of the same kind in optical lattices, less is known about multi-species models where there are different types of atoms or atoms with internal degrees of freedom. An experimentally relevant multi-species model is the Bose-Hubbard model where bosons carry an internal spin-1 degree of freedom. This model describes unpolarized ${ }^{23} \mathrm{Na}$ atoms having nuclear spin-1 in optical traps. The Hamiltonian is

$$
H=-t \sum_{<i j>}\left(a_{i \sigma}^{\dagger} a_{j \sigma}+\text { H.c. }\right)+\frac{U}{2} \sum_{i} n_{i}\left(n_{i}-1\right)+\frac{U_{2}}{2} \sum_{i} \vec{S}_{i} \cdot \vec{S}_{i},
$$

$\vec{S}_{i}$ is a spin-1 operator and $n_{i}$ is the boson density at site $i$.

This Hamiltonian conserves the number of particles, and so the DMC evolution will also keep the particle number constant. By measuring the energy difference between the different particle number sectors one can obtain the chemical potential at the boundaries of the Mott insulating regions with particle densities $\alpha$ for a lattice with $L$ sites as

$$
\begin{aligned}
& \mu_{+}(\alpha)=E(\alpha L+1)-E(\alpha L) \\
& \mu_{-}(\alpha)=E(\alpha L)-E(\alpha L-1)
\end{aligned}
$$

where $E(N)$ is the ground state energy of the system with $N$ particles. The phase boundaries between the Mott insulator and the superfluid is shown in Fig. 2 for $U_{2} / U=0.2$. The plot was obtained by extrapolating finite size values for $\mu_{ \pm}$for $\alpha=1,2$. In contrast to the single-species Bose-Hubbard model where the second Mott lobe is smaller than the first, the antiferromagnetic interaction helps stabilize the second Mott lobe over a bigger region in the phase diagram. This is due to the fact that the antiferromagnetic interaction lowers the energy of two particles on the same site. This energy gain is significant in the second Mott lobe, but not in the first as there is only one particle per site. One should note that magnetic transitions might be present within the Mott lobes and that the superfluid can also be of an exotic variant ${ }^{6}$. We have focused solely on particle density variables here, thus Fig. 2 contains no direct information about magnetic structures. 


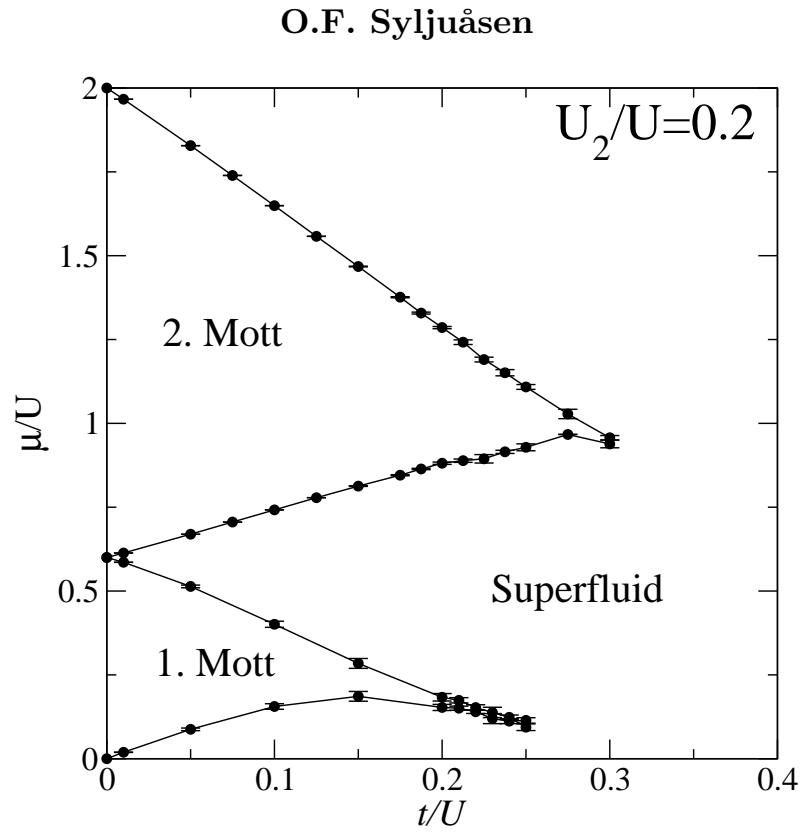

Fig. 2. Phase boundaries between Mott insulating regions and superfluid for $U_{2} / U=0.2$ for a $1 \mathrm{D}$ chain. The points are obtained by linearly extrapolating to $L=\infty$ from system sizes $L=8,16,20,24$.

\section{QUANTUM DIMER MODEL}

The quantum dimer model $(\mathrm{QDM})^{7}$ is the simplest model capturing the essentials of the Resonant Valence Bond(RVB) scenario for antiferromagnets ${ }^{8}$ where spins form singlets. In the QDM the singlets are modeled as dimers which fully cover the lattice without overlaps, see Fig. 3. The QDM assigns a potential energy $V$ to plaquettes having parallel dimers, and a resonance energy $J$ is associated to flips in the orientation of parallel dimers around a plaquette. The QDM can be extended by including doped monomers, where essentially one dimer is replaced with two monomers which can hop around

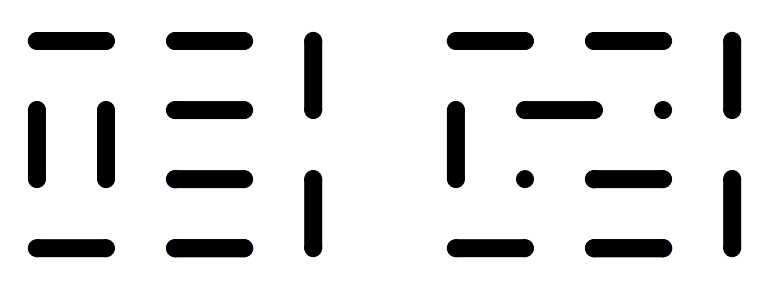

Fig. 3. Snapshots of an undoped and a doped dimer configuration. 


\section{Continuous-time Diffusion Monte Carlo Algorithm for Lattice Models}

independently with hopping strength $t$. The Hamiltonian is

$$
\begin{aligned}
H= & -J \sum(|\mathbf{I}\rangle\langle\mathbf{=}|+\text { H.c. })+V \sum(|\mathbf{I}\rangle\langle\mathbf{I}|+| \mathbf{I}|\langle\mathbf{=}|) \\
& -t \sum_{\langle i j\rangle,\langle j k\rangle}(|\bullet-\rangle\langle\mathbf{- \bullet}|+\text { H.c. })
\end{aligned}
$$

The two first sums are taken over all elementary plaquettes of the square lattice. In the third sum, $i$ and $j$ are nearest neighbors as are $j$ and $k$, allowing the monomer to move "around corners" as well as moving "straight".

The ground state of the QDM can be found exactly at $J=V, t=0$, the so called Rokhsar-Kivelson(RK) point ${ }^{9}$. It is well known that performance of DMC is drastically improved by guiding the random walkers using a good trial wave function. Here we will use the exact solution at the RK-point as trial wave function for all $V$. Implementing this is extremely easy. This is because the exact solution at the RK-point is the equal-amplitude superposition of all basis states (dimer states). When no explicit trial wave function is specified in DMC, the equal-amplitude superposition of all basis states is the implicit choice made, thus one can proceed as if one didn't include any explicit trial wave function!

\subsection{Undoped quantum dimer model}

In the $V=J$ ground state on the square lattice dimer-dimer correlation functions decay as power laws, and no ordered dimer pattern exists. A longstanding question is whether or not this lack of ordering also exists for $V<J$. Exact diagonalizations performed for system sizes up to $8 \times 8$ have concluded that it does not ${ }^{10,11}$. We have investigated this for significantly larger system sizes by studying the columnar order parameter

$$
\chi_{\mathrm{col}}^{2}=\frac{1}{4 N^{2}}\left\langle\left(\sum n_{\mathrm{H}}(\vec{r})(-1)^{r_{x}}\right)^{2}+\left(\sum n_{\mathrm{V}}(\vec{r})(-1)^{r_{y}}\right)^{2}\right\rangle
$$

where $n_{\mathrm{H}}\left(n_{\mathrm{V}}\right)$ is the number of horizontal (vertical) dimers belonging to the plaquette centered around $\vec{r}$. Fig. 4 shows a plot of the columnar order parameter as a function of $V / J$ measured using the forward-walking technique $^{12}$. It supports the conclusion that the columnar order exists all the way up to $V=J$.

Columnar order implies confinement of monomers. This is because the introduction of monomers necessarily breaks up the ordered arrangement in between monomers. Thus the energy cost increases with monomer separation. A direct way of measuring this energy cost is to measure the groundstate energy in the presence of static $(t=0)$ monomers. Doing this for 
O.F. Syljuåsen

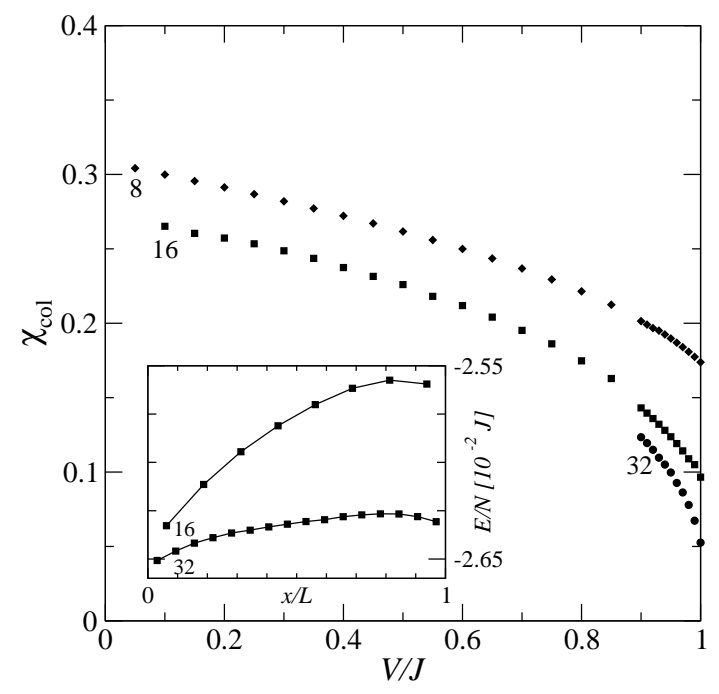

Fig. 4. Columnar order parameter vs. $V / J$ for different system sizes. Inset: The ground-state energy of the QDM at $V / J=0.9$ with two static monomers displaced a distance $\mathrm{x}$ for two system sizes $L=16,32$.

different distances between the monomers one obtains the energy curves shown in the inset of Fig. 4. It is seen that the energy keeps increasing with distance, thus monomers are confined.

\subsection{Doped quantum dimer model}

The DMC algorithm allows also the study of the doped quantum dimer model. It is interesting to know at which values of doping or monomer hopping strengths the columnar order is destroyed. This would signal a monomer deconfinement transition. In Fig. 5 we have plotted the columnar order parameter vs. inverse linear system size for different values of $t$ at fixed values of $V / J$ and doping. It is seen that the columnar order parameter extrapolates to finite values for $t<t_{c} \approx 0.2$ and extrapolates to zero for bigger values. For $t>t_{c}$ we thus expect deconfined monomers. Inset is an energy-curve showing the energy of two static monomers displaced a distance $x$ in a background of dimers and dynamic monomers at $V / J=0.9$ and $t / J=1$. This curve exhibits a maximum for $x<L / 2$ thus indicating deconfinement of monomers.

Repeating this for other doping concentrations $n$ and values of $V / J$ we obtain the phase boundary shown in Fig. 6 . 
Continuous-time Diffusion Monte Carlo Algorithm for Lattice Models

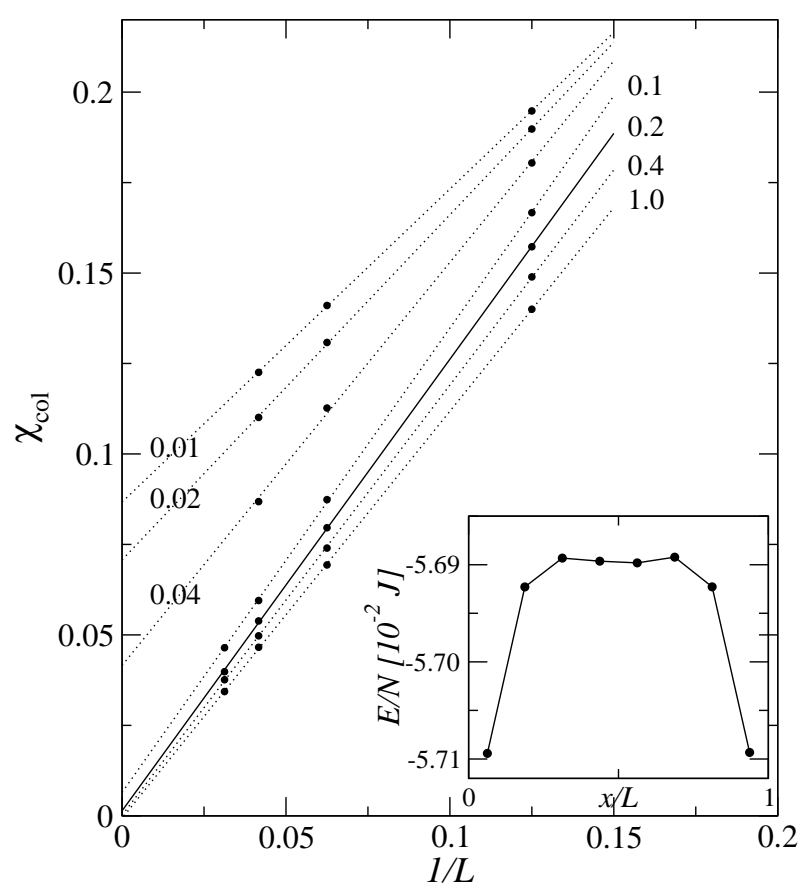

Fig. 5. Columnar order parameter vs inverse linear system size for different values of $t$ at $V / J=0.9$ and doping $n=1 / 32$. Inset: The ground-state energy for a system with two static monomers displaced a distance $\mathrm{x}$ for $t / J=1, V / J=0.9$ and $n=1 / 32$.

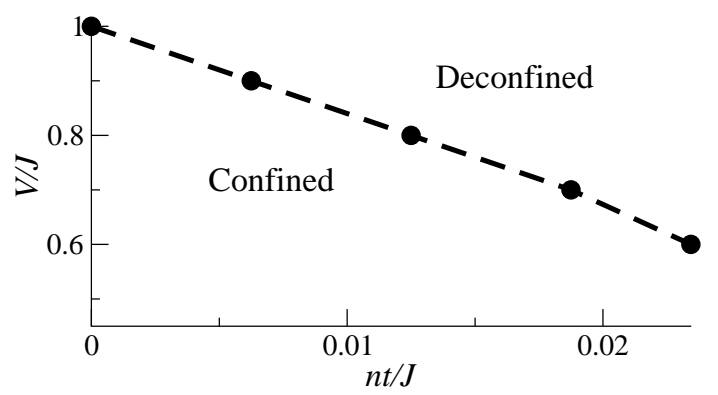

Fig. 6. Phase boundary between the monomer confined and deconfined phases in the doped quantum dimer model. 


\section{O.F. Syljuåsen}

\section{CONCLUSIONS}

The Diffusion Monte Carlo method can be formulated in continuous imaginary time for lattice models. It is a good tool to study lattice models at zero temperature and is to a large degree complimentary to Monte Carlo methods that samples the finite-temperature partition function ${ }^{13}$. We have applied it to two quite different models and obtained the lowest Mott lobes in the spin-1 antiferromagnetic Bose-Hubbard model as well as shown the

existence of deconfined monomers in the square lattice doped quantum dimer model.

\section{REFERENCES}

1. for a review and further refs. see D. Ceperley, B. Alder, Science, 231, 555 (1986).

2. O. F. Syljuåsen, Phys. Rev. B 71, 020401(R) (2005).

3. C. J. Umrigar, M. P. Nightingale, and K. J. Runge, J. Chem. Phys. 99, 2865 (1993).

4. B. B. Beard and U.-J. Wiese, Phys. Rev. Lett. 77, 5130 (1996).

5. M. Greiner et al., Nature 415, 39 (2002).

6. E. Demler and F. Zhou, Phys. Rev. Lett. 88, 163001 (2002).

7. S. A. Kivelson, D. S. Rokhsar and J. P. Sethna, Phys. Rev. B 35, 8865 (1987).

8. P. Fazekas and P. W. Anderson, Philos. Mag. 30, 23 (1974).

9. D. S. Rokhsar and S. A. Kivelson, Phys. Rev. Lett. 61, 2376 (1988).

10. S. Sachdev, Phys. Rev. B 40, 5204 (1989).

11. P. W. Leung, K. C. Chiu and K. J. Runge, Phys. Rev. B 54, 12938 (1996).

12. K.S. Liu, M.H. Kalos, and G.V. Chester, Phys. Rev. A 10, 303 (1974).

13. O. F. Syljuåsen and A. W. Sandvik, Phys. Rev. E 66, 046701 (2002). 